\title{
Producción de textos mediante entornos educativos virtuales del software Ardora: Una experiencia de aplicación en pandemia
}

Recepción: 21/03/2021 | Revisión: 25/04/2021 | Aceptación: 07/12/2021 | Publicación: 01/03/2022

\section{Cristopher David HERRERA NAVAS}

Pontificia Universidad Católica

del Ecuador - Sede Santo Domingo

cdherreran@pucesd.edu.ec

https://orcid.org/oooo-0002-2031-5187

en tiempos de COVID-19

\author{
Gabriela Ibeth SALTOS PIGUAVE \\ Pontificia Universidad Católica \\ del Ecuador - Sede Santo Domingo \\ gisaltosp@pucesd.edu.ec \\ https://orcid.org/oooo-0002-5570-2099
}

iD Edgar Efraín OBACO SOTO

Pontificia Universidad Católica

del Ecuador - Sede Santo Domingo

osef@pucesd.edu.ec

https://orcid.org/oooo-0002-7243-5869

Resumen: En la actualidad se ha identificado que la mayor parte de estudiantes del contexto ecuatoriano producen textos con escasa originalidad, sin coherencia, con errores gramaticales y dificultades para la expresión de ideas personales. El objetivo de la presente investigación fue mejorar la producción de textos mediante estrategias didácticas virtuales en el software Ardora. La metodología de estudio responde a un enfoque cuantitativo con un diseño cuasiexperimental, de tipo transversal con alcance descriptivo. Se aplicó una prueba diagnóstica para evaluar las habilidades de redacción de 56 estudiantes del subnivel de Básica Media ${ }^{1}$. Los resultados demuestran que la muestra posee un nivel de dominio bajo para producir textos, siendo el indicador más afectado las reglas gramaticales. Sin embargo, al utilizar estrategias didácticas en el software Ardora, se alcanzó un nivel medio en las dimensiones siguientes: tipos de texto, estructura básica del texto, propiedades textuales y producción de textos personales. Finalmente, a través de la prueba de rangos de Wilcoxon, se acepta la hipótesis de investigación planteada $\mathrm{H} 1$, concluyendo que el uso de estrategias didácticas virtuales en el software Ardora mejora la producción de textos escritos.

Palabras clave: estrategias educativas; informática educativa; redacción; educación básica.

\footnotetext{
${ }^{1}$ Básica Media: $5^{\circ}, 6^{\circ}$ y $7^{\circ}$ grado de Educación General Básica, generalmente se ofrece a estudiantes de 9 a 11 años de edad (Ministerio de Educación del Ecuador, 2016).
} 


\title{
TEXT PRODUCTION USING ARDORA SOFTWARE IN VIRTUAL EDUCATIONAL EN- VIRONMENTS: AN APPLICATION EXPERIENCE DURING THE PANDEMIC
}

\begin{abstract}
It has been identified that most students in the Ecuadorian context currently produce texts with little originality, without coherence, with grammatical errors and difficulties in the expression of personal ideas. The objective of this research was to improve the production of texts through virtual didactic strategies in the Ardora software. This is a quantitative study with a quasi-experimental, cross-sectional design, and with a descriptive scope. A diagnostic test was applied to evaluate the writing skills of 56 students of the middle school level. The results show that the sample has a low level of mastery to produce texts, being the most affected indicator the grammatical rules. However, by using didactic strategies in the Ardora software, a medium level was achieved in the following dimensions: text types, basic text structure, textual properties and personal text production. Finally, through the Wilcoxon rank test, the research hypothesis H1 is accepted, concluding that the use of virtual didactic strategies in the Ardora software improves the production of written texts.
\end{abstract}

Key words: educational strategies; ICT tools; drafting; basic education.

\section{PRODUCCIÓ DE TEXTOS MITJANÇANT ENTORNS EDUCATIUS VIRTUALS DEL SOFT- WARE ARDORA: UNA EXPERIÈNCIA D'APLICACIÓ DURANT LA PANDÈMIA}

\begin{abstract}
Resum: S'ha identificat que la major part d'estudiants del context equatorià produeixen actualment textos amb escassa originalitat, sense coherència, amb errors gramaticals i dificultats per a l'expressió d'idees personals. L'objectiu de la present recerca va ser millorar la producció de textos mitjançant estratègies didàctiques virtuals en el programari Ardora. La metodologia d'estudi respon a un enfocament quantitatiu amb un disseny quasi-experimental, de tipus transversal amb abast descriptiu. Es va aplicar un test diagnòstic per a avaluar les habilitats de redacció de 56 estudiants del subnivell de Bàsica Mitjana. Els resultats demostren que la mostra posseeix un nivell de domini baix per a produir textos, sent l'indicador més afectat les regles gramaticals. No obstant això, en utilitzar estratègies didàctiques en el programari Ardora, es va aconseguir un nivell mitjà en les dimensions següents: tipus de text, estructura bàsica del text, propietats textuals i producció de textos personals. Finalment, a través de la prova de rangs de Wilcoxon, s'accepta la hipòtesi de recerca plantejada H1, concloent que l'ús d'estratègies didàctiques virtuals en el programari Ardora millora la producció de textos escrits.
\end{abstract}

Paraules clau: estratègies educatives; informàtica educativa; redacció; educació bàsica.

\section{Introducción}

La producción de textos escritos es una función cognitiva superior que permite la inserción académica y social del individuo, y constituye un medio de comunicación sociocultural reglamentado ya que la síntesis de la cultura es el lenguaje. Sin embargo, en la actualidad se ha identificado que la mayor parte de estudiantes del contexto producen textos con escasa originalidad, sin coherencia, con faltas ortográficas, errores gramaticales y dificultades para la expresión de ideas personales (Herrera et al., 2020).

La problemática relacionada con el pobre desarrollo de habilidades para la producción de textos es un tema de interés global. Así lo señala la Organización de las Naciones Unidas para 
la Educación Ciencia y Cultura [UNESCO] (2015), afirmando que los estudiantes a nivel mundial carecen de habilidades de redacción, escribiendo con escasa originalidad, sin coherencia y con faltas de ortografía. Complementariamente, se menciona que la causa principal es el mal uso de los recursos y la aplicación de metodologías que no responden a las necesidades de los alumnos.

Otro estudio que brinda una perspectiva actual del escollo a nivel de Latinoamérica es El Tercer Estudio Regional Comparativo y Explicativo [TERCE] (2015), donde se señala que existen dificultades en la producción de textos escritos a nivel regional. Es así que, a través de una evaluación aplicada a 15 países, obtienen como resultados que, en una escala valorativa de $1-4$ puntos, Colombia, Honduras, Paraguay, República Dominicana y Ecuador están por debajo de la media.

De manera más específica, en el contexto ecuatoriano, también existe evidencia de la dificultad en los estudiantes para producir textos escritos, así lo señalan Herrera y Olmedo (2020), quienes determinan que los estudiantes presentan serios problemas para la redacción relacionados con las normas ortográfica, gramaticales y la expresión de textos personales. También se menciona que el factor pedagógico prima en la generación de los problemas para la producción de textos escritos, ya que los docentes no utilizan estrategias metodológicas adecuadas, no aplican de forma correcta los niveles de concreción curricular y no se brinda una atención individualizada a los estudiantes.

De manera análoga, como problemática educativa y social, en el año 2020 surge el virus SARS-CoV-2 (COVID-19), que provoca una pandemia que obliga a todos los países a implementar protocolos de aislamiento y distanciamiento social. Es así que, en todos los niveles de educación, se impone de forma necesaria el trabajo virtual, por lo cual surge la necesidad de buscar nuevas alternativas metodológicas que favorezcan el proceso educativo.

Haciendo referencia a la problemática expuesta, existen diversas investigaciones que destacan la importancia de desarrollar la producción de textos en los alumnos a partir de alternativas innovadoras. A nivel internacional, Arroyo $(2015$, p. 6) realiza en España un estudio denominado "La escritura creativa en el aula de educación primaria". Teniendo como objetivo utilizar la técnica de escritura creativa para la producción de textos, con un enfoque mixto, transversal y de tipo descriptivo. Los resultados del trabajo arrojan que los docentes no están preparados o no tienen los recursos para aplicar esta nueva técnica. Sin embargo, es una actividad que puede contribuir a mejorar la producción de textos en el aula. Se concluye que los métodos y estrategias que se utilizan actualmente para desarrollar la producción de textos escritos se centran en la adquisición de la mecánica para escribir y no en el progreso de la expresión escrita.

Desde la misma línea de investigación, enfatizando en la lectura como uno de los elementos indispensables para la producción de texto escritos, se encuentra Granja (2018, p. 1). Su investigación denominada "Diseño e implementación de estrategia pedagógica en el grado 3-b a través de herramientas TIC (Ardora)" tiene el objetivo de diseñar e implementar estrategias pedagógicas mediante el uso de Ardora a través de una metodología de enfoque mixto con diseño cuasiexperimental de tipo descriptivo. Como principales resultados de la investigación antes mencionada, los autores obtienen que el uso de Ardora promueve el aprendizaje significativo, motivando y generando implicación de los estudiantes, que prefieren las TICs sobre la educación transmisora de información. Se concluye que el uso de Ardora tuvo un alto impacto en la mejora de la lectura de los estudiantes, dado que el 95,75\% de la muestra alcanzó los objetivos. 
Como último antecedente, desde el contexto ecuatoriano, López (2016, p. 7) analiza la problemática en su estudio "La motivación por parte del docente en el área de Lengua y Literatura y la construcción de textos escritos" con el propósito de establecer la incidencia de la motivación del educador en la construcción de textos de los alumnos. Obtiene como resultado que gran parte de discentes no logran producir un texto con claridad y precisión, debido a que los docentes aplican escasas estrategias para el desarrollo de esta destreza. El autor concluye que la motivación y el uso de estrategias didácticas adecuadas por parte del educador son esenciales para la correcta elaboración de textos escritos.

Los estudios presentados anteriormente hacen referencia a las dificultades de la producción de textos escritos, formas de partida para su tratamiento y la incorporación de las TICs como herramienta de apoyo. Esto se ha indagado por pedagogos desde dimensiones psicológicas y pedagógicas; sin embargo, a nivel contextual, no se ha propuesto el uso del programa Ardora como estrategia desde la dimensión didáctica para desarrollar la producción de textos escritos de los estudiantes del subnivel de básica media. Por ello, el objetivo general de la investigación fue: mejorar la producción de textos escritos mediante estrategias didácticas virtuales en el software Ardora aplicadas en estudiantes del subnivel de Básica Media. Asimismo, los objetivos específicos fueron: a) Diagnosticar el nivel de desarrollo del dominio de producción de textos escritos; b) Diseñar una propuesta de intervención basada en estrategias didácticas virtuales en el software Ardora para la mejora de la producción de textos escritos; c) Aplicar estrategias didácticas virtuales en Ardora para la mejora de la producción de textos escritos; d) Evaluar los resultados obtenidos a partir de la implementación de estrategias didácticas virtuales en Ardora para la mejora de la producción de textos escritos.

\section{Definición de estrategia didáctica}

Las estrategias didácticas son fundamentales dentro del proceso educativo y son aquellas que, dependiendo de su elección y eficacia, pueden dar resultados positivos en el aprendizaje de los estudiantes. Bravo y Varguillas (2015) la definen como una serie de actividades que se determinan con base en los contenidos y ayudan a la consecución de los objetivos establecidos dentro de la clase. Estas actividades se realizan de manera intencional con la finalidad de guiar el aprendizaje en los discentes de forma significativa.

Asimismo, Zúñiga (2017) menciona que las estrategias didácticas son procedimientos que tienen una secuencia y organización que están orientadas a la consecución de las metas previamente establecidas dentro de la clase. Estas actividades son seleccionadas por los profesores, quienes están en la responsabilidad de detallarlas y diseñarlas en base a las necesidades de los estudiantes, por lo que su elección será de forma reflexiva y consiente con el propósito de alcanzar los objetivos.

Por otro lado, Díaz (2019) señala que las estrategias didácticas pueden ser consideradas modelos de innovación que pueden utilizar los docentes si logran identificar las necesidades de los alumnos y con ello puede que logren implementar en el salón de clases estrategias didácticas que puedan mejorar la calidad de los aprendizajes y generar en los estudiantes una motivación que facilitará el proceso educacional.

Se puede considerar que las estrategias didácticas constituyen una secuencia de actividades que tienen un procedimiento y un orden lógico durante las clases. Estas son determinadas 
por los docentes con el propósito de favorecer la formación de los discentes. Para determinar las estrategias didácticas, es necesario que los profesores tengan en cuenta las necesidades de los estudiantes, los contenidos a tratar y los objetivos que se quieren lograr.

\subsection{Fases de aplicación de las estrategias didácticas}

Las fases para el desarrollo de estrategias didácticas contribuyen con una orientación del proceso que se debe tener en cuenta para su implementación. Tienen como finalidad obtener como resultado un óptimo aprendizaje en los estudiantes. Según Cárdenas et al. (2018) y Carrera (2012) las fases de aplicación de las estrategias metodológicas son: modelación, práctica guiada, práctica independiente y se las define de la siguiente manera: a) modelación: es la fase 1 de la aplicación de las estrategias didácticas y consiste en el que el profesor da a conocer cada paso de la estrategia didáctica teniendo como finalidad explicar las fases que se seguirán tanto desde el ámbito procedimental como conceptual, facilitando la construcción del conocimiento; b) práctica guiada: es la fase 2, que radica en el desarrollo de la estrategia didáctica con la guía y acompañamiento del docente, aquí se presentan dos fases que son, 1) guía explicita que la realizan los profesores y 2 ) auto-instrucción por parte de los estudiantes; c) práctica independiente: es la fase 3 de la aplicación de las estrategias didácticas que busca beneficiar a los discentes por medio de experiencias y situaciones que permitan la práctica y aplicación del conocimiento obtenido.

\subsection{Importancia de las estrategias didácticas}

Las estrategias didácticas cumplen un papel importante dentro del ámbito educativo, debido a que colaboran significativamente con el proceso de enseñanza-aprendizaje. Su importancia, según Ramos (2016), radica en que son propuestas innovadoras, no tradicionalistas, que permiten que los estudiantes puedan llegar a dominar habilidades, destrezas, competencias y que los aprendizajes que adquieran puedan aplicarse durante la vida.

Del mismo modo, se puede decir que la importancia de las estrategias didácticas se centra en la facilitación y la potenciación del proceso educativo de los discentes, basado en los principios de individualización y ritmos de aprendizaje de cada estudiante, tomando en cuenta, principalmente el estilo cognitivo de cada uno. Si se aplica de manera adecuada las estrategias didácticas tomando en cuenta lo señalado los resultados serán favorables y se podrá conseguir los objetivos planteados (Hurtado, 2016).

En consonancia con lo antes mencionado, Aventura (2015) menciona que la significación de las estrategias didácticas surge porque es necesario que los alumnos lleven a cabo nuevos procesos de aprendizaje que se alejen de las formas clásicas de enseñanza, donde se les limitaba únicamente a memorizar; a través de la aplicación de las estrategias se busca que el estudiante indague, investigue para ser el protagonista de su proceso de enseñanza-aprendizaje.

Se puede establecer que las estrategias didácticas son un pilar fundamental en el ámbito educativo que favorece el aprendizaje de los discentes. A través de ellas, se pueden lograr los objetivos establecidos en clase; en este caso, a través del uso de las estrategias didácticas, se pretende mejorar la producción de textos de los estudiantes de octavo año de Educación General Básica (EGB). 


\subsection{Estrategias didácticas virtuales}

Las estrategias didácticas se aplican de diferente modo, según el entorno del proceso educativo. En este caso, los entornos considerados son los virtuales, por lo tanto, es necesario definir qué son las estrategias didácticas virtuales. Sanza (2018) señala que son aquellas que se aplican dentro de ambientes virtuales que explican y orientan a los alumnos en las actividades que deben ejecutar, con la finalidad de alcanzar los objetivos planteados previamente.

De la misma manera, Carrillo et al. (2019) mencionan que las estrategias didácticas virtuales son aquellas que generan motivación y realzan el interés de los discentes en el aprendizaje y la comprensión de los distintos temas que se imparten en clases virtuales. Se considera que la implementación de estrategias didácticas virtuales favorece el desarrollo de la implicación y la creatividad de los estudiantes, debido que, permiten generar aprendizaje más interactivos y dinámicos con la finalidad de completar el proceso de enseñanza-aprendizaje.

Por otro lado, se establece que las estrategias didácticas virtuales son aquellas que consideran los docentes para ambientes virtuales y se aplican a través de herramientas web, lo cual hace que se basen en el constructivismo social. Las estrategias didácticas virtuales se escogen a partir del contenido que se quiere impartir en clases y tomando en cuenta en las necesidades de cada uno de los discentes, por lo cual, es necesario tener en cuenta las teorías de aprendizaje al momento de seleccionarlas (Sanza, 2016).

En la actualidad se necesita conocer las estrategias didácticas virtuales y cómo poder implementarlas en el proceso educativo. A partir de lo anterior, se puede decir que las estrategias didácticas virtuales son aquellas que se aplican en entornos virtuales que, pese su gran diferencia respecto del ambiente presencial, tienen la misma finalidad: contribuir al proceso de enseñanza-aprendizaje y alcanzar los objetivos planteados.

\subsection{Ardora}

Existen diversas herramientas en la web que pueden utilizarse para favorecer el proceso educativo, sin embargo, en el presente trabajo investigativo se ha seleccionado Ardora para mejorar la producción de texto de los estudiantes del 8 vo año de EGB, por lo tanto, es necesario definirla. Según Garzón et al. (2019, p. 3) "Ardora es una herramienta que brinda la facilidad de crear contenido de índole educativo y que, a su vez, puedan ser compartidos a través de la web sin depender de expertos en programación y diseño".

De igual manera, Muñoz et al. (2018) señalan que Ardora es una herramienta donde los docentes pueden crear en formato HTML más de 35 actividades de diversos tipos, entre estas: simetrías, esquemas, paneles gráficos, páginas multimedia como: zooms de imágenes, galerías y reproductores $\mathrm{mp} 3$ y $\mathrm{mp} 4$. También permite crear páginas para servidor, las cuales permiten la elaboración de líneas de tiempo, póster, álbum colectivo, entre otras que favorecen el trabajo colaborativo.

Por su parte, Góngora (2015, p. 17) menciona que "Ardora es una aplicación informática para docentes que permite crear sus propios contenidos web, de un modo muy sencillo, sin tener conocimientos técnicos de diseño o programación web". Es una herramienta que facilita el proceso educativo, debido a que permite que los docentes únicamente se preocupen en incluir los elementos necesarios en cada actividad, también cabe mencionar que esta aplicación es de uso gratuito y solamente se necesita un navegador para poder divisar su contenido. 
En consonancia con las definiciones anteriores de Ardora, se puede establecer que es una herramienta eficaz que puede favorecer positivamente el aprendizaje de los estudiantes, ya que cuenta con variedad de actividades, orientadas a contribuir con los diferentes temas que se trabajan en el ámbito educativo, por lo tanto, seleccionando de manera correcta las actividades también puede llegar a favorecer la producción de textos de los estudiantes de $8{ }^{\circ}$ año de EGB, que es lo que se pretende con este trabajo.

\section{Definición y características de la producción de textos escritos}

La conceptualización de producción de textos escritos posee diversas definiciones, en función de los elementos enfatizados y áreas estudiadas. Para la consolidación de la temática, se considera preciso definir el tema desde varios enfoques y concluir con los aspectos en común. Es así como Ríos (2012) lo define como un proceso cognitivo de complejidad que consiste en plasmar, a través de un discurso coherente, respetando un conjunto de normas y con un enfoque contextual comunicativo, un mensaje determinado que comprende ideas, sentimientos, pensamientos e impresiones de todo tipo propios del sujeto. Desde esta perspectiva, el texto se convierte en un medio comunicativo sociocultural reglamentado.

Del mismo modo, se especifica que existen normas que regulan la producción de textos. Entre ellas se encuentran: "aspectos de ortografía, de uso de léxico, de arreglo sintáctico, de comunicación de significados, de estilo y organización textual. Producción de ideas en forma escrita en función de audiencias específicas, con ciertas intenciones comunicativas y dentro de contextos y prácticas comunicativas concretas" (Ríos, 2012, p. 96).

Por otro lado, con algunos puntos en común con el autor antes abordado, se encuentran Olaya y Villamil (2012), para los que la redacción de textos escritos y la producción de textos escritos son procesos idénticos, que a diferencia de escribir, que consiste en el mero acto de plasmar trazos a partir de un código, estos constituyen un código completo de expresión del pensamiento mediante la escritura (que pasa a ser herramienta de la producción de textos escritos) conformada por habilidades básicas de la escritura (propiedades textuales) mediante una estructura, normativa y organización adecuada en función de la intencionalidad comunicativa que se le otorgue. Todos esos elementos y habilidades son apropiados mediante la lectura y la práctica procedimental. Entonces, se puede considerar la producción de textos escritos como una habilidad cognitiva compleja que, a través de la escritura, permite expresar pensamientos, ideas, sentimientos y vivencias de forma estructurada, mediante niveles con una función, respetando diversas normas de la comunicación escrita y en función de un contexto comunicativo.

\subsection{Estándares de aprendizaje orientados a la producción de textos escritos}

Con el afán de regular y mantener la calidad de la educación, el Ministerio de Educación del Ecuador $(2012$, p. 6) establece estándares de calidad educativa que "son descriptores de logros esperados correspondientes a los diferentes actores e instituciones del sistema educativo. En tal sentido, son orientaciones de carácter público que señalan las metas educativas para conseguir una educación de calidad". Es decir, presentan los estados ideales de cada una de las áreas implicadas en el proceso institucionalizado de formación humana (el aprendizaje, la gestión escolar, el desempeño profesional y la infraestructura). 
En este apartado, se hará énfasis en los estándares de aprendizaje que describen los logros de los estudiantes que en una educación de calidad deberían alcanzar en cada etapa de su formación académica, iniciando en la EGB y culminando en el Bachillerato. Estos estándares se encuentran divididos en las cuatro áreas básicas del saber (Ciencias Naturales, Estudios Sociales, Matemáticas y Lengua y Literatura) y organizados por dominios y niveles que poseen relación progresiva en sus resultados. En el caso del presente apartado, se hará énfasis en los estándares de aprendizaje del área de Lengua y Literatura, puesto que, en ellos se aborda la producción de textos escritos.

Para comprender mejor la organización de los estándares de aprendizaje, se necesita describir cada uno de los elementos internos. Entre ellos, se encuentran los dominios del conocimiento que son ejes centrales de aprendizaje de la ciencia y que abordan de forma independiente cada área curricular. Su intencionalidad es abarcar todos los elementos de cada ámbito mediante sus aspectos principales (Ministerio de Educación del Ecuador, 2012).

El área de Lengua y Literatura cuenta con los dominios: "a) Comunicación oral; b) Comprensión de textos escritos; c) Producción de textos escritos" (Ministerio de Educación del Ecuador, 2012, p. 5). Cada uno de ellos organiza los estándares que se deben cumplir en cada área. Cabe recalcar que el currículo sirve de herramienta para el desarrollo de destrezas con criterio de desempeño que permitan al estudiante, al finalizar un rango determinado de niveles, cumplir con los estándares de aprendizaje que establecen los dominios. Otro de los elementos que organizan los estándares de aprendizaje son los niveles de progresión, cada uno corresponde a un rango de organización de los estándares de aprendizaje, comprendiendo una serie de elementos de desarrollo que se dan de forma procesual a lo largo de la vida escolar del estudiante. En el caso del presente trabajo, se hará énfasis en los estándares condensados en el dominio c (producción de textos escritos) correspondientes al tercer nivel, que comprende el subnivel de básica media. Es decir, los estudiantes, al culminar el 7.0 año de EGB, tendrán que contar con una serie de habilidades que se expondrán a continuación.

\subsection{Estándares del tercer nivel del dominio C producción de textos escritos}

Ya teniendo clara la definición de estándares de aprendizaje, los elementos que los organizan (niveles y dominios) y el área en la que enfatiza el presente trabajo, se considera preciso establecer de forma literal los estándares que forman parte del tercer nivel, del dominio c producción de textos escritos, los cuales son:

Produce textos escritos, literarios y no literarios, para exponer o informar. Organiza ideas sobre un tema central y considera la estructura de un texto. Utiliza las partes de un párrafo introductorio y conclusivo. Emplea un vocabulario variado y mantiene la concordancia verbal; aplica las normas ortográficas básicas y el uso correcto de los signos de puntuación. Escribe textos sobre experiencias personales y diversas situaciones cotidianas, con propósitos comunicativos específicos. (Ministerio de Educación del Ecuador, 2012, p. 23)

\subsection{Producción de textos mediado por tecnologías digitales}

Cada vez es mayor el campo que abarcan las tecnologías digitales, influyendo en la mayor parte de áreas en las que se desenvuelve el ser humano. Uno de los ámbitos en el que se ha vinculado y continúa evolucionando con nuevas tendencias, es la educación, donde se han implementado propuestas para el desarrollo de diversas funciones cognitivas, campo que abarca a la producción de textos. Berdugo et al. (2010) mencionan que se requiere de innovación, funcionalidad y 
entretenimiento para que un estudiante aprenda a producir textos escritos. Sin embargo, la competencia debe ser puesta en práctica en diversos contextos.

De la misma forma, Callejas y Méndez (2019) señalan que, para la utilización de entornos virtuales que favorezcan el mejoramiento de la producción de textos escritos de los estudiantes, es necesario que haya una previa planificación por parte del docente, mediante la cual se debe seleccionar una herramienta virtual que se ajuste a las necesidades de los discentes. Asimismo, se sugiere una constante revisión del proceso para los ajustes necesarios según los requerimientos estudiantiles. Estos entornos virtuales deben caracterizarse por permitir elaborar estrategias innovadoras fundamentadas en los postulados del aprendizaje significativo, con la finalidad de que los estudiantes no consideren la producción de textos como un acto motriz, sino como la capacidad de poder comunicar ideas y pensamientos de manera adecuada.

Existen diversas estrategias que pueden llegar a utilizar los docentes para mejorar las habilidades de redacción, como el uso de entornos virtuales que permitan crear actividades individuales, grupales y colectivas, las cuales favorecerán el entusiasmo y la motivación de los estudiantes por aprender. La importancia de esta habilidad radica en ampliar y enriquecer la competencia escritora de los estudiantes debido a que esta les ayudará a poder desenvolverse en el día a día y en su vida profesional (Silva, 2019).

De igual manera, se considera preciso que para el desarrollo de la competencia para producir textos escritos en entornos virtuales de aprendizaje, se opte por utilizar plataformas que brinden la capacidad de adaptarse al estudiante, trabajar desde varias inteligencias y ofrecer actividades desde la dinámica del juego. Esto posibilitará la generación de condiciones que requiere el aprendizaje significativo y no se generarán solo aprendizajes memorísticos. Otra recomendación que se brinda, en este sentido, es que los docentes se capaciten en el dominio de entornos virtuales de aprendizaje, ya que la transpolación de contenidos a saber enseñable en dichos entornos suele tener ciertas características que precisan de conocimiento básico sobre las opciones que existen (Enríquez, 2011).

A partir del contraste de la problemática abordada referente a las dificultades para producir textos escritos y la revisión teórica de las estrategias didácticas virtuales, se propone establecer las siguientes hipótesis de investigación: H1: Ardora como estrategia didáctica virtual mejora la producción de textos escritos de los estudiantes. H0: Ardora como estrategia didáctica virtual no mejora la producción de textos escritos de los estudiantes.

\section{Materiales y métodos}

El supuesto metodológico del presente estudio está conformado por un enfoque cuantitativo, con un diseño cuasiexperimental, donde se manipuló la variable independiente para poder observar su consecuencia en otra, es decir, la dependiente. En este sentido, la investigación posee tres momentos, que son: 1 ) pretest: se verificó, a través una evaluación, el estado inicial del grupo en torno al fenómeno a estudiar; 2) se aplicó la propuesta de intervención, que constituye la variable independiente, para observar su efecto (mejora o permanencia del problema) en la variable dependiente; 3 ) postest: se repitió el instrumento de evaluación del pretest para verificar el estado final de los sujetos luego de aplicar la intervención. De esta forma, se procedió a calcular el porcentaje de mejora en cada uno de los indicadores que conforman las 
dimensiones de las variables (Hernández et al., 2014). La investigación es de tipo descriptiva, permitiendo así caracterizar cualidades y particularidades del fenómeno en su realidad natural.

La población de estudio es de 756 estudiantes que conforman la EGB de la Unidad Educativa Gonzalo Pizarro en el año lectivo 2020. Como muestra, se seleccionó por conveniencia a 56 estudiantes de los paralelos: A (26) y B (30), teniendo en cuenta dos criterios que responden a los intereses de la investigación: a) deben pertenecer a octavo año de EGB, ya que, en este nivel los estudiantes deben alcanzar los estándares que plantea el área de Lengua y Literatura en el nivel tres del dominio c, producción de textos escritos; b) aquellos grupos que presentan dificultades en la producción de textos escritos.

El instrumento utilizado para la recolección de datos (pretest y postest) fue propuesto por Herrera y Olmedo (2019) en un estudio similar que abordó la variable producción de textos desde todas sus dimensiones, desarrollado en el mismo contexto de educación básica, con ejercicios de desarrollo orientados al diagnóstico de las habilidades para la producción de textos. Además, cuenta con una validación por criterio de expertos que garantizan la claridad y pertinencia de sus ítems.

Para la recolección de resultados, se solicitó la autorización de la autoridad competente, además del consentimiento de los padres de familia de la muestra estudiada para la aplicación del instrumento durante el pretest, postest y la propuesta de intervención en Ardora. Las actividades se realizaron desde la virtualidad. Finalmente, para analizar los datos, se utilizó la estadística descriptiva (que permitió realizar cálculos de las medidas de tendencia central) e inferencial, a través de la cual se realizó la prueba de hipótesis.

\section{Análisis y resultados \\ 4.1 Primer resultado}

A continuación, se describen los resultados del pretest que responde al primer objetivo específico de la investigación que fue: diagnosticar el nivel de desarrollo del dominio de producción de textos escritos de los estudiantes. Para ello, se utilizó la escala del Ministerio de Educación (2016) que se presenta en la Tabla 1:

\begin{tabular}{clc}
\hline Escala cuantitativa & \multicolumn{1}{c}{ Escala cualitativa } & Nivel de desarrollo \\
\hline $9,00-10,00$ & Domina los aprendizajes requeridos & Alto \\
$7,00-8,99$ & Alcanza los aprendizajes requeridos & Medio \\
$4,01-6,99$ & Está próximo a alcanzar los aprendizajes requeridos & \\
$\leq 4$ & No alcanza los aprendizajes requeridos & Bajo \\
\hline
\end{tabular}

Tabla 1. Escala cualitativa y cuantitativa del Ministerio de Educación. Fuente: adaptado del Ministerio de Educación del Ecuador (MINEDUC) (2012). ${ }^{2}$

\footnotetext{
${ }^{2}$ De acuerdo con el Ministerio de Educación del Ecuador (2016) la escala cualitativa de evaluación puede ser utilizada con las siguientes siglas: DAAR: domina los aprendizajes requeridos PAAR: próximo a alcanzar los aprendizajes requeridos AAR: alcanza los aprendizajes requeridos 
Puede observarse en la Tabla 2 que el diagnóstico del nivel de desarrollo de todas las dimensiones que conforman la producción de textos escritos es bajo. Destacan las reglas gramaticales, donde obtuvieron el puntaje más bajo $(3,07)$, seguido de la producción de textos personales $(5,07)$. Conforme a la escala cualitativa, los estudiantes se encuentran, en la mayoría de las dimensiones, próximos a alcanzar los aprendizajes requeridos, aunque en una dimensión no alcanzan los aprendizajes requeridos.

Esto denota que la muestra presenta serios problemas con el manejo de las reglas gramaticales, de manera específica en las normas ortográficas básicas y los signos de puntuación. Es decir, presentan dificultades en tildar correctamente las palabras y el uso correcto de los signos de puntuación.

Del mismo modo, se puede evidenciar conflictos con el correcto uso de las propiedades textuales, donde los textos redactados carecen de coherencia, cohesión y adecuación; la estructura básica de un texto, debido que no se redacta respetando introducción, desarrollo y conclusión; la redacción atendiendo a un tipo de texto y la producción de textos escritos personales con una intencionalidad comunicativa específica.

\begin{tabular}{lccc}
\hline \multicolumn{1}{c}{ Dimensión } & \multicolumn{2}{c}{ PRETEST } & Nivel de desarrollo \\
\hline Tipos de texto & 5,08 & Pscala & BAJO \\
Estructura básica del texto & 5 & PAAR & BAJO \\
Propicdades textualcs & 5,33 & PAAR & BAJO \\
Reglas gramaticales & 3,07 & NAAR & BAJO \\
Producción de textos personales & 5,07 & PAAR & BAJO \\
\multicolumn{1}{c}{ TOTAL } & 4,71 & PAAR & BAJO \\
\hline
\end{tabular}

Tabla 2. Contraste entre escalas de evaluación y niveles de desarrollo. Fuente: adaptado MINEDUC (2012).

Esto denota también que la muestra presenta serios problemas con el manejo de las reglas gramaticales, de manera específica en las normas ortográficas básicas y los signos de puntuación. Es decir, presentan dificultades en: tildar correctamente las palabras y en el uso correcto de los signos de puntuación.

\subsection{Segundo resultado: propuesta de intervención}

En el presente apartado, se presenta la propuesta de intervención que da cumplimiento al segundo objetivo específico, que es: diseñar una propuesta de intervención de estrategias didácticas virtuales en el software Ardora para la mejora de la producción de textos escritos de los estudiantes.

La propuesta está estructurada sobre la base de cinco estrategias didácticas virtuales que atienden a cada dimensión evaluada. Cada estrategia didáctica consta de seis plantillas de actividades que se elaboraron en formato HTML en Ardora. La composición de cada estrategia se encuentra diseñada a partir de algunos elementos del modelo estructural de estrategias didácticas gamificadas propuesto por Herrera y Veloz (2020), los cuales son: a) mecanismos 
rectores: objetivo concreto; b) mecanismos desarrolladores: constituyen algunas funciones didácticas centrales (motivación, aseguramiento del nivel de partida, tratamiento del nuevo contenido, fijación y consolidación); c) mecanismos de control: hacen referencia a la forma de verificar el nivel de cumplimiento de los objetivos (control y valoración del rendimiento).

De igual manera, las estrategias didácticas virtuales planteadas se sustentan en los postulados del aprendizaje significativo propuesto por David Ausubel, específicamente en los siguientes: 1) Los conocimientos previos y los prerrequisitos son elementos imprescindibles para la construcción del nuevo conocimiento; 2) Las estrategias implementadas deben partir desde el análisis de la circunstancia actual de los estudiantes y las capacidades intelectuales que poseen; 3) La motivación es fundamental, el estudiante debe querer aprender; 4) Toda estrategia didáctica implementada debe ayudar a buscar y recolectar un conocimiento que evidencie su utilidad (Ausubel, 2002).

Los pasos para crear actividades en formato HTML, una vez se tenga descargado Ardora, se pueden resumir del siguiente modo: 1) abrir Ardora; 2) seleccionar plantilla de actividades a utilizar; 3) configurar plantilla con los datos del ejercicio, instrucciones y formato estético de la página; 4) generar archivo HTML; 5) crear paquete de actividades enlazadas. A continuación, en las Tablas 3, 4 y 5, se presenta una síntesis de las estrategias didácticas virtuales utilizadas, con la descripción de las actividades:

\begin{tabular}{|c|c|}
\hline $\begin{array}{l}\text { Dimensión: tipos de texto. } \\
\text { Objetivo: producir textos literarios y no literarios para } \\
\text { exponer o informar comprendiendo su uso y } \\
\text { funcionalidad individual. }\end{array}$ & $\begin{array}{l}\text { Dimensión: estructura básica del texto. } \\
\text { Objetivo: interiorizar la estructura básica del texto } \\
\text { produciendo textos escritos que organicen ideas de forma } \\
\text { correcta. }\end{array}$ \\
\hline $\begin{array}{l}\text { Estrategia didáctica virtual. } \\
\text { - Motivación: armar el rompecabezas siguiendo la } \\
\text { secuencia para descubrir la temática a abordar en la } \\
\text { estrategia didáctica virtual. } \\
\text { - Conocimientos previos: resolver la sopa de letras a } \\
\text { través de las pistas proporcionadas estableciendo los } \\
\text { requisitos necesarios para acceder al nuevo } \\
\text { conocimiento. } \\
\text { - Prerrequisitos: clasificar en organizadores gráficos } \\
\text { los textos literarios y no literarios comprendiendo su } \\
\text { uso y funcionalidad individual. } \\
\text { - Tratamiento del nuevo contenido: producir textos } \\
\text { literarios y no literarios atendiendo a su estructura y } \\
\text { propósito comunicativo a través de la pizarra virtual } \\
\text { comprendiendo su uso y funcionalidad individual. } \\
\text { - Fijación y consolidación: interiorizar la temática } \\
\text { abordada a través de la lectura y visualización de } \\
\text { imágenes referente a los textos literarios y no } \\
\text { literarios contribuyendo a la mejora de su uso y } \\
\text { comprensión de la funcionalidad individual. } \\
\text { - Control y valoración del rendimiento: etiquetar } \\
\text { textos de ejemplo como literarios y no literarios según } \\
\text { corresponda demostrando la apropiación del tema. }\end{array}$ & $\begin{array}{l}\text { Estrategia didáctica virtual. } \\
\text { - Motivación: armar el rompecabezas siguiendo la secuencia } \\
\text { para descubrir la temática a abordar en la estrategia didáctica } \\
\text { virtual. } \\
\text { - Conocimientos previos: resolver la sopa de letras a través de } \\
\text { las pistas proporcionadas estableciendo los requisitos } \\
\text { necesarios para acceder al nuevo conocimiento. } \\
\text { - Prerrequisitos: clasificar en organizadores gráficos la } \\
\text { estructura básica de un texto a partir de los elementos } \\
\text { establecidos reconociendo cada elemento de la estructura } \\
\text { básica de un texto. } \\
\text { - Tratamiento del nuevo contenido: producir textos } \\
\text { respetando su estructura básica (introducción, desarrollo y } \\
\text { conclusión) a través de la pizarra virtual reconocimiento cada } \\
\text { elemento de la estructura básica de un texto. } \\
\text { - Fijación y consolidación: interiorizar la temática abordada a } \\
\text { través de la lectura y visualización de imágenes referentes a } \\
\text { la estructura básica de un texto contribuyendo a la } \\
\text { ejercitación y apropiación de los elementos necesarios para la } \\
\text { correcta producción de textos escritos. } \\
\text { - Control y valoración del rendimicnto: unir con líncas las } \\
\text { partes de la estructura básica de un texto con el texto que } \\
\text { corresponde, contribuyendo a la ejecución y apropiación de } \\
\text { los elementos necesarios para la correcta producción de } \\
\text { textos escritos. }\end{array}$ \\
\hline
\end{tabular}

Tabla 3. Planificación de la primera y segunda estrategia didáctica. 


\begin{tabular}{ll}
\hline $\begin{array}{l}\text { Dimensión: propiedades textuales. } \\
\text { Objetivo: comprender las propiedades textuales, } \\
\text { contribuyendo a la producción de textos escritos } \\
\text { coherentes, cohesionados y adecuados al contexto. }\end{array}$ & $\begin{array}{l}\text { Dimensión: reglas gramaticales. } \\
\text { Objetivo: aplicar las normas ortográficas básicas y los } \\
\text { signos de puntuación, contribuyendo con la correcta } \\
\text { producción de textos escritos. }\end{array}$ \\
\hline \multicolumn{1}{c}{ Estrategia didáctica virtual. } & \multicolumn{1}{c}{ Estrategia didáctica virtual. } \\
- Motivación: armar el rompecabezas siguiendo la & - Motivación: armar el rompecabezas siguiendo la \\
secuencia para descubrir la temática a abordar en la & secuencia para descubrir la temática a abordar cn la \\
estrategia didáctica virtual. & estrategia didáctica virtual.
\end{tabular}

Tabla 4. Planificación de la tercera y cuarta estrategia didáctica.

Dimensión: producción de textos personales

Objetivo: produce textos escritos sobre experiencias personales, contribuyendo a la expresión escrita de situaciones cotidianas a través de la producción de texto escritos.

Estrategia didáctica virtual

- Motivación: armar el rompecabezas siguiendo la secuencia para descubrir la temática a abordar en la estrategia didáctica virtual.

- Conocimientos previos: resolver la sopa de letras a través de las pistas proporcionadas estableciendo los requisitos necesarios para acceder al nuevo conocimiento.

- Prerrequisitos: clasificar en organizadores gráficos los propósitos comunicativos de la producción de textos personales a través de los recursos facilitados contribuyendo con la expresión personal a través de la producción de textos escritos.

- Tratamiento del nuevo contenido: producir textos escritos personales con propósitos comunicativos específicos a través de la pizarra virtual contribuyendo a la expresión personal a través de la producción de textos escritos. - Fijación y consolidación: interiorizar la temática abordada a través de la lectura y visualización de imágenes referentes a la expresión personal en la producción de textos escritos con propósitos comunicativos específicos. - Control y valoración del rendimiento: realizar un cómic que relate una experiencia personal a través de los recursos facilitados contribuyendo a la expresión personal a través de la producción de textos escritos.

Tabla 5. Planificación de la quinta estrategia didáctica. 


\subsection{Tercer resultado: aplicación de la propuesta}

En el presente apartado, se abordan los resultados procedentes de la aplicación de la propuesta de intervención, dando cumplimiento al objetivo de aplicar estrategias didácticas virtuales en Ardora para la mejora de la producción de textos escritos. Los datos que se presentan en la Tabla 6 reflejan que en la ejecución de la propuesta de intervención a partir de Ardora como estrategia didáctica, los estudiantes se posicionan mayormente en un nivel de dominio medio en la producción de textos escritos, únicamente variando en la dimensión de reglas gramaticales, donde, pese a mejorar en relación con el pretest que se encontraba en la escala de NAAR, obtienen 6,36 de promedio general, reflejando según la escala que se encuentran próximos a alcanzar los aprendizajes requeridos y que aún poseen un nivel de dominio bajo de la dimensión.

\begin{tabular}{lccc}
\hline \multicolumn{1}{c}{ Dimensión } & \multicolumn{2}{c}{ EJECUCIÓN DE LA PROPUESTA } & \\
& Promedio & Escala & Nivel de desarrollo \\
\hline Tipos de texto & 7,40 & AAR & MEDIO \\
Estructura básica del texto & 7,34 & AAR & MEDIO \\
Propiedades textuales & 7,43 & AAR & MEDIO \\
Reglas gramaticales & 6,36 & PAAR & BAJO \\
Producción de textos personales & 7,60 & AAR & MEDIO \\
\multicolumn{1}{c}{ TOTAL } & 7,23 & AAR & MEDIO \\
\hline
\end{tabular}

Tabla 6. Resultados de ejecución de la propuesta.

Esto representa que las estrategias didácticas virtuales en Ardora han apoyado en mayor medida con la mejora de las dificultades relacionadas con las propiedades textuales, el uso correcto de la tilde y signos básicos de puntuación, la concepción de estructura básica de un texto y el reconocimiento de los tipos de texto existentes por sus características, asimismo, se verifica una mejora en la producción de textos escritos personales.

\subsection{Cuarto resultado: postest}

Los datos que se observan en la Tabla 7 reflejan los resultados obtenidos en el postest. Se puede visualizar, de manera similar que en la ejecución de la propuesta, que los estudiantes se posicionan mayormente en un nivel de dominio medio para la producción de textos escritos, variando de forma única en la dimensión referente a reglas gramaticales, donde obtienen 6,51 de promedio general, que pese a constituir un avance significativo en relación al promedio del pretest $(3,07)$ aún refleja según la escala del Ministerio de Educación que se encuentran próximos a alcanzar los aprendizajes requeridos.

Por ende, se verifica que las estrategias didácticas virtuales implementadas en Ardora han apoyado en el mejoramiento de las dificultades relacionadas con la producción de texto escritos, especialmente el reconocimiento de los tipos de texto, en la concepción de estructura básica de un texto y en la compresión de las propiedades textuales, sin embargo, no hay que dejar de mencionar que se necesita seguir trabajando con la dimensión cuatro que corresponde a las reglas gramaticales. 


\begin{tabular}{lccc}
\hline & \multicolumn{2}{c}{ POSTEST } & \\
\multicolumn{1}{c}{ Dimensión } & Promedio & Escala & Nivel de desarrollo \\
\hline Tipos de texto & 7,46 & AAR & MEDIO \\
Estructura básica del texto & 7,40 & AAR & MEDIO \\
Propiedades textuales & 7,51 & AAR & MEDIO \\
Reglas gramaticales & 6,51 & PAAR & BAJO \\
Producción de textos personales & 7,67 & AAR & MEDIO \\
\multicolumn{1}{c}{ TOTAL } & 7,31 & AAR & MEDIO \\
\hline
\end{tabular}

Tabla 7. Resultados del postest.

\subsection{Comprobación de hipótesis}

En función de los resultados obtenidos en el pretest y postest, se procedió a aplicar la prueba no paramétrica de Wilcoxon para muestras relacionadas, con la finalidad de comprobar la hipótesis de investigación o aceptar la hipótesis nula (ver Tabla 8). El cálculo del estadístico demuestra en primera instancia que no existen rangos negativos, es decir, ningún estudiante bajó su promedio. También se pudo identificar que los resultados del postest son mayores a los del pretest, ya que la totalidad de estudiantes obtienen promedios que se ubican en rangos positivos de mejora. Como consecuencia de ello, se obtiene una significancia asintótica bilateral de 0,000 que resulta <0,05, en función del estadístico de Wilcoxon se acepta la hipótesis de investigación $\mathrm{H} \_1$, por lo cual, se puede decir que Ardora como estrategia didáctica virtual mejora significativamente la producción de textos escritos.

\begin{tabular}{llr}
\hline & \multicolumn{1}{c}{ Prueba de rangos del estadístico de Wilcoxon } \\
\hline POS_TEST - & Rangos negativos & $0^{\mathbf{a}}$ \\
PRE_TEST & Rangos positivos & $56^{\mathbf{b}}$ \\
& Empates & $0^{\mathbf{c}}$
\end{tabular}

a. POS_TEST $<$ PRE_TEST

b. POS TEST $>$ PRE TEST

c. POS_TEST $=$ PRE_TEST

Sig. asintótica(bilateral)

0,000

Tabla 8. Prueba de Wilcoxon para comprobación de hipótesis.

\section{Discusión}

Como primer resultado se diagnostica que los estudiantes poseen un nivel de desarrollo bajo en todas las dimensiones que conforman la producción de textos escritos, resultados similares obtienen Herrera y Olmedo (2020) en su investigación sobre las causas que dificultan la redacción, donde los estudiantes del subnivel de básica media presentan problemas con el correcto uso las normas ortográficas, gramaticales y de acentuación. Sin embargo, en cuanto a propiedades textuales, el autor presenta un porcentaje de error de $37,8 \%$, que no supera la media de $70 \%$, según lo detalla la investigación. Esto se debe a que el autor evaluó las propiedades textuales por separado, sin considerar su cohesión en la redacción. No obstante, Espinoza (2009), en su investigación de las propiedades textuales a partir del desarrollo de textos, determina que el $80 \%$ de la muestra no las utiliza de forma correcta. De manera análoga Sotomayor et al. 
(2018) concuerdan con los resultados obtenidos por el presente estudio, coincidiendo con Herrera y Olmedo (2020) y Espinoza (2009) en que algunos de los problemas más evidentes son la carencia de tildes, errores en la ubicación grafías y organización del texto.

Las dificultades para producir textos escritos antes mencionadas, se pueden atribuir a lo que plantea la UNESCO (2015) sobre la deficiente formación de los docentes en el manejo de recursos y estrategias metodológicas - para enseñar a redactar- acorde a las necesidades de los alumnos. De igual manera, se podría deber a lo mencionado por Herrera et al. (2020) acerca de que los docentes no brindan una atención individualizada, carecen de un proceso didáctico y sistematizado, con continuidad para enseñar a producir textos escritos y no enfatizan en la semántica de las palabras cuando enseñan a redactar, esto ocasiona dificultades con el uso de las propiedades textuales y las normas que rigen la escritura (ortografía y gramática).

A esto, se suman diversos factores contextuales como el pedagógico, ya que los docentes han demostrado, en ciertos casos, que no tienen claros cuáles son los propósitos de aprendizaje que debe lograr el discente conforme a lo establecido en la desagregación de objetivos de aprendizaje de los niveles de concreción curricular. Del mismo modo, no se atienden a las necesidades educativas especiales en el aula de clase, es común presenciar el abandono pedagógico en los procesos de enseñanza de la redacción.

En cuanto al segundo resultado, se diseña una propuesta de intervención basada en la aplicación de estrategias didácticas con el software Ardora, estructurada a partir de las cinco dimensiones que conforman la producción de textos escritos: tipos de texto, estructuración básica del texto, propiedades textuales, reglas gramaticales y producción de textos personales. En cada estrategia se elabora un objetivo y se añaden seis plantillas de actividades que responden a las funciones didácticas, elementos que concuerdan con el modelo estructural de estrategias didácticas gamificadas propuesto por Herrera y Veloz (2020) donde se agrupan todos los componentes y se los categoriza como mecanismos rectores (objetivo), mecanismos desarrolladores (funciones didácticas de desarrollo), mecanismos de control (funciones didácticas de control y valoración). De forma similar, Granja (2018), en su estudio para dinamizar el aprendizaje en el área de español, plantea estrategias pedagógicas en la herramienta Ardora direccionando las actividades a partir de indicadores de logro establecidos y añadiendo plantillas de actividades con el siguiente orden: actividades de ambientación, actividades de aseguramiento del nivel de partida, actividades de desarrollo y actividades de aplicación o cierre. La propuesta mencionada y su diseño interno también concuerda con los postulados del aprendizaje significativo de Ausubel (2002), donde se considera necesario definir las características de partida del alumnado y su capacidad cognitiva, usar los conocimientos previos que ya posee, considerar la motivación como elemento imprescindible y enfocar los aprendizajes hacia la determinación de su utilidad.

Esto pretende responder a la carencia determinada en el contexto, donde se aplican técnicas y estrategias enfocadas al desarrollo de la producción de textos escritos aislando cada uno de sus componentes, es decir, se enseña ortografía, gramática, propiedades textuales, estructuración y tipos de texto, pero no se articulan los elementos de forma lógica y secuencial para desarrollar las habilidades básicas que le permitan al estudiante producir textos. Como consecuencia de esto, los estudiantes no logran alcanzar los niveles de dominio propuestos por los estándares de aprendizaje del Ministerio de Educación del Ecuador (2012) en el área de Lengua y Literatura. 
Esto obliga a repensar en nuevas técnicas o estrategias basadas en la tecnología que permitan mejorar los procesos de enseñanza y aprendizaje en la producción de textos escritos.

Como tercer resultado se aplicó la propuesta de intervención basada en Ardora, la cual obtuvo como resultado que los estudiantes se posicionen mayormente en un nivel de dominio medio en la producción de textos escritos, sin embargo, existe una variación en la dimensión de reglas gramaticales, donde pese a la mejora -en relación a los resultados originarios del diagnóstico inicial-, los estudiantes siguen teniendo un nivel de dominio bajo con un promedio general de 6,36. Resultados similares presenta Martínez (2015) en su estudio, donde en la prueba de diagnóstico obtuvo como resultado que el $100 \%$ de los estudiantes presentan errores ortográficos en dictados y en la producción de textos escritos debido al escaso vocabulario que poseen, por lo que se concluye que es la causa que afecta la redacción y la composición de textos escritos. De forma similar, en el estudio antes mencionado, a partir de la aplicación de herramientas virtuales para la mejora de la ortografía, los resultados reflejan que los estudiantes mejoraron luego de desarrollar diversas actividades correspondientes a la producción de textos escritos, sin embargo, los discentes aún cuentan con un vocabulario limitado, repetición de palabras en una misma frase, cantidad de palabras incorrectas escritas con $\mathrm{H}$ e uso inadecuado de los signos de puntuación.

Parcialmente en concordancia con el presente trabajo investigativo de tipo transversal, se encuentra la investigación de López y Rodríguez (2017), quienes plantean que para tener resultados significativos, se necesita un tiempo de seis meses de implementación del programa y un seguimiento longitudinal, sin embargo, se concuerda con estos autores en que Ardora es una herramienta que cumple con el objetivo de apoyar los diferentes problemas para la producción de textos escritos. Asimismo, Romero (2015) indica que el uso de Ardora mejora el proceso de enseñanza-aprendizaje, lo cual se fundamenta en sus resultados, donde el $68 \%$ de los estudiantes mejoraron significativamente.

En el último resultado se evaluó el impacto de la implementación de estrategias didácticas virtuales con Ardora mediante un postest que registró un nivel de dominio medio; por lo tanto, se confirma que las estrategias didácticas virtuales empleadas en Ardora han mejorado significativamente la producción de texto escritos, específicamente en el reconocimiento de los tipos de texto, la concepción de estructura básica de un texto y la compresión de las propiedades textuales. De manera análoga, Berdugo et al. (2010) concuerdan a partir de su estudio, que la implementación de estrategias didáctica virtuales mejoran la producción de textos escritos, debido a que, a partir de sus resultados se constató que se facilita la adquisición competencias de argumentación y el aprendizaje del uso de conectores lógicos, reglas ortográficas, construcción de frases, estructura de un texto y la adquisición de vocabulario. De manera similar, Jaramillo (2014) señala que a partir de herramientas virtuales se pueden generar estrategias didácticas que contribuyan a la mejora de la producción de textos escritos, porque considera que generan un enriquecimiento de los procesos educativos.

En función del progreso obtenido, se presume que las herramientas tecnológicas generan un nivel de motivación significativo que promueve la asimilación significativa de los contenidos trabajados por los estudiantes, en este caso, el desarrollo de las habilidades para la producción de textos escritos (Carrillo et al., 2019). Además, se debe considerar que al ser Ardora una herramienta intuitiva y fácil de manejar no implica dificultades mayores en el desarrollo de las actividades (Garzón et al., 2019). 


\section{Conclusiones}

Se diagnosticó que el nivel de desarrollo del dominio de producción de textos escritos de los estudiantes es bajo, presentando dificultades con el empleo correcto de las normas ortográficas básicas y los signos de puntuación, la producción de textos personales, la estructuración básica del texto (introducción, desarrollo y conclusión), la producción de textos respetando el tipo solicitado (literarios y no literarios) y el respeto de las propiedades textuales (coherencia, cohesión y adecuación). Esto permite inferir que al culminar el subnivel de básica media, los estudiantes no alcanzan el nivel tres de los estándares de aprendizaje propuestos como descriptores de logro por el Ministerio de Educación del Ecuador, lo que representa un problema significativo ya que la producción de textos es determinante en la sociedad actual, vista como herramienta de inserción académica y social. La percepción, comprensión y comunicación de la cultura que rodea al ser humano se encuentra sintetizada por el lenguaje escrito.

A partir del diseño de la propuesta de intervención enfocada en la mejora de producción de textos escritos mediante de Ardora como estrategia didáctica virtual, se pudo percibir que, para elaborar estrategias didácticas virtuales funcionales, es necesario realizar un diagnóstico de las capacidades para producir textos escritos de los alumnos y con ello, plantear objetivos acordes a sus necesidades. También se precisa la elección de un modelo estructural de actividades que organice la secuencia didáctica de forma lógica y contribuya al cumplimiento de los objetivos. Es decir, no se debe recaer en la improvisación no planificada. Además, cada actividad debe encontrarse enfocada en el estudiante y la destreza que se plantea desarrollar.

Al aplicar Ardora como estrategia didáctica virtual, se pudo constatar que los estudiantes lograron un nivel de dominio medio, excepto en la dimensión de reglas gramaticales, donde siguen teniendo un nivel de dominio bajo. Por tanto, se demuestra que Ardora es una herramienta que sirve para mejorar la producción de textos escritos. No obstante, es recomendable utilizar un tiempo mayor de aplicación para trabajar todas las dimensiones, lo cual posibilitará que los estudiantes lleguen aún nivel de dominio alto y mejoren el uso de las normas gramaticales, permitiendo que los discentes tengan un adecuado uso de las reglas ortográficas, entre estas: una escritura correcta de las palabras, una adecuada tildación y el uso correcto de los signos de puntuación.

En cuanto a la evaluación del impacto de la propuesta de intervención, se tuvo como resultado que los estudiantes alcanzaron un nivel de dominio medio; por tanto, se puede concluir que el impacto de la experimentación fue positivo debido a que Ardora es una herramienta que se ajusta a las circunstancias actuales de la educación y es generadora de motivación e interés en el aprendizaje de los estudiantes, por lo que se recomienda continuar aplicando esta herramienta para el mejoramiento de la producción de textos escritos y obtener así un dominio alto de esta destreza. 


\section{Referencias}

Arroyo, R. (2015). La escritura creativa en el aula de educación primaria. [Tesis de pregrado, Universidad de Cantabria]. https://bit.ly/2EM3iFj

Ausubel, D. (2002). Adquisición y retención del conocimiento. Una perspectiva cognitiva. Paidós.

Aventura, C. (2015). Estilos de aprendizaje y prácticas de enseñanza en la universidad. Un binomio que sustenta la calidad educativa. Perfiles Educativos, 33(1), 142-154. https://bit.ly/32IuoqK

Berdugo, M., Herrera, O. y Valdiri, V. (2010). El desarrollo de la escritura académica en el ambiente virtual Lingweb: realidades y desafíos. Lenguaje, 38(2), 351-386. https://doi.org/gw94

Bravo, P. y Varguillas, C. (2015). Estrategias didácticas para la enseñanza de la asignatura Técnicas de Estudio en la Universidad Nacional de Chimborazo. Sophia, Colección de Filosofía de la Educación, 1(19), 271-290. https://bit.ly/3g3T7ut

Callejas, J., y Méndez, M. (2019). Estrategias didácticas basadas en un objeto virtual de aprendizaje para apoyar los procesos de comprensión lectora [Tesis de maestría, Universidad Cooperativa de Colombia]. https://bit.ly/3pcJ5eH

Cárdenas, S., Ríos, M. y Rentería, H. (2018). Los organizadores previos, una estrategia de comprensión lectora para asimilar la nueva información de textos informativos en el área de lengua castellana. Un estudio en básica primaria en la Institución Educativa Manuela Beltrán de Yopal Casanare. [Tesis de maestría, Universidad de la Salle]. https://bit.ly/3gFXR7V

Carrera, J. (2012). Uso de diagramas de flujo y sus defectos en la enseñanza - aprendizaje de contenidos procedimentales. [Tesis doctoral, Universitat de Lleida]. https://bit.ly/2EFP4Gf

Carrillo, S., Tigre, F., Tubón, E. y Sánchez, D. (2019). Objetos Virtuales de Aprendizaje como estrategia didáctica de enseñanza aprendizaje en la educación superior tecnológica. Recimundo, 3(1), 287-304. https://doi.org/gw93

Díaz, A. (2019). Estudio experimental sobre estrategias didácticas innovadoras y tradicionales en la enseñanza de Estudios Sociales. Revista Electrónica de Conocimientos, Saberes y Prácticas, 2(1), 21-35. https://doi.org/g8mz

Enríquez, S. (2011). La enseñanza de la escritura en entornos virtuales. Puertas Abiertas, 1(7), 1-9. https://bit.ly/3wPJPcl

Espinoza, E. (2009). Comunicación: principales dificultades. Escritura y pensamiento, 12(25), 85-100. https://bit.ly/39EtCgF

Garzón, M., Rosado, M. y Bello, Y. (2019). Los objetos de aprendizajes como una alternativa en el proceso de enseñanza-aprendizaje. Espacios, $40(36), 1-8$. https://bit.ly/3pDvGNB

Góngora, R. (2015). Aplicación de software educativo Ardora en el proceso de enseñanza aprendizaje de las Ciencias Naturales, en la Unidad Educativa Fiscomisional "1o de agosto" [Tesis de maestría]. https://bit.ly/31BsyHc

Granja, M. (2018). Diseño e implementación de estrategia pedagógica en el grado 3-b a través de herramientas TIC (Ardora) para dinamizar el proceso de enseñanza y aprendizaje en el área de español. [Tesis de posgrado, Universidad Nacional de Colombia]. https://bit.ly/3lwzxZJ

Hernández, R., Fernández, C. y Baptista, M. (2014). Metodología de la investigación. https://bit.ly/3gDlMFe

Herrera, C. y Olmedo, R. (2019). Causas que dificultan la redacción en los estudiantes del subnivel de básica media. [Tesis de pregrado, Pontificia Universidad Católica del Ecuador]. https://bit.ly/3ltihor

Herrera, C., Olmedo, R. y Obaco, E. (2020). Causas que dificultan la redacción de textos. Digital Publisher CEIT, 5(4), 24-37. https://doi.org/gw92 
Herrera, C. y Veloz, N. (2020). Modelo estructural de estrategias didácticas gamificadas: una perspectiva re-dinamizadora del proceso de enseñanza-aprendizaje desde las TICs. [Resumen de presentación de la ponencia]. En G. E. Cevallos, Y. Ramos, A. Alcivar y L. Jácome (Eds.), $1 .^{\circ}$ Congreso tecnológico COTEC-202O.

Hurtado, G. (2016). Las estrategias didácticas activas en el aprendizaje de la resolución de problemas de química. Influencia del estilo cognitivo del estudiante. Tecné, Episteme y Didaxis, 1(39), 31-51. https://doi.org/gw9z

Jaramillo, I. (2014). Los ambientes virtuales de aprendizaje: herramientas de apoyo para el trabajo independiente del estudiante en los cursos de lectoescritura de la USTA. [Tesis de pregrado, Universidad Santo Tomas]. https://ng.cl/oeovd

López, F. (2016). La motivación por parte del docente en el área de Lengua y Literatura y la construcción de textos escritos. [Tesis de grado, Universidad Técnica de Ambato]. https://bit.ly/31EN9uc

López, L. y Rodríguez C. (2017). Ardora como recurso educativo digital para el fortalecimiento de competencias ambientales de los niños y niñas de grado segundo del Ied Atabanzha. [Tesis de pregrado, Fundación Universitaria Los Libertadores]. https://bit.ly/3AxLftK

Martínez, Y. (2015). Uso de páginas virtuales para el fortalecimiento de la ortografía del español. [Tesis de pregrado, Universidad Libre]. https://n9.cl/ho7sv

Ministerio de Educación del Ecuador (2012). Estándares de calidad educativa. https://bit.ly/3lszVuE

Ministerio de Educación del Ecuador. (2016). Educación General Básica. https://bit.ly/3akAlwA

Ministerio de Educación del Ecuador. (2016). Instructivo: Aplicación de la evaluación estudiantil. https://bit.ly/3AxLcy4

Muñoz Carril, P. C., Fuentes Abeledo, E. J. y Álvarez de Sotomayor, I. D. (2018). El diseño de cuentos interactivos multimedia a través de "EdiLIM": Análisis de una experiencia en el Grado de Educación Infantil (pp. 324-331). En D. Gonçalves, J. Fernández Rodríguez, M. Castro Rodríguez, M. C. Montserrat, Ricoy Lorenzo, J. Rodríguez Rodríguez y X. M. Cid Fernández (Orgs.), A Fenda Dixital. TIC, NEAE, Inclusión e Equidade. https://bit.ly/3lxJMNo

Olaya, M. y Villamil, N. (2012). La producción de textos como recurso didáctico para la escritura creativa. [Tesis de pregrado, Universidad Libre]. https://bit.ly/2YJl4js

Ramos, E. (2016). Estrategias didácticas en la enseñanza-aprendizaje: lúdica en el estudio de la nomenclatura química orgánica en alumnos de la Escuela Preparatoria Regional de Atotonilco. Educación Química, 27(1), 43-51. https://doi.org/g8m2

Ríos, D. (2012). Comprensión y producción de textos escritos a través del aprendizaje colaborativo en estudiantes Universidad Manizales. Escribania, 10(2), 93-99. https://bit.ly/39EtCgF

Romero, B. (2015). El software educativo ardora como herramienta de aprendizaje significativo en los estudiantes de la Unidad Educativa "Ocho de Noviembre" de la parroquia Balsapamba de la provincia de Bolívar 2015 - 2016. [Tesis de pregrado, Universidad Técnica de Babahoyo]. https://bit.ly/3iaWgLR

Sanza, I. (2016). Estrategias didácticas en tecnologías web para ambientes virtuales de aprendizaje. Praxis, 12(1), 1-8. https://doi.org/g8m3

Sanza, I. (2018). Propuesta didáctica para ambientes virtuales de aprendizaje desde el enfoque praxeológico. Praxis \& Saber, 9(20), 1-21. https://doi.org/gw9x

Sepulveda, C. y Moreno, L. (2017). Análisis de la inversión pública aplicada al desarrollo sustentable al Municipio de Puerto Peñasco, Sonora. El Periplo Sustentable, 32(32), 82-109. https://ng.cl/vnwel

Silva, E. (2019). Proceso de producción textual en un taller de escritura virtual para estudiantes de quinto grado. Revista Panorama, 13(25), 51-58. https://bit.ly/3paAPfg 
Sotomayor, C., Bedwell, D., Molina, P. y Hernández, C. (2018). Caracterización de problemas ortográficos recurrentes en alumnos de escuelas municipales chilenas de $3 \cdot{ }^{\circ}, 5 \cdot{ }^{\circ}$ y $7 \cdot{ }^{\circ}$ básico. Revista Signos, 46(81), 105-131. https://doi.org/g8m4

TERCE (2015). Análisis curricular. https://bit.ly/3gFdzjP

UNESCO (2015). El arte de la escritura. https://bit.ly/3b9KM5M

Zúñiga, M. (2017). La estrategia didáctica: Una combinación de técnicas didácticas para desarrollar un plan de gestión de riesgos en la clase. Revista Educación, 41(1), 1-18. https://doi.org/gw95 